\title{
KINEMATICS OF GALACTIC GLOBULAR CLUSTERS FROM
}

\section{SCHMIDT-PLATE ASTROMETRY}

New results for $M 5$ and $M 12$

\author{
R.-D. SCHOLZ ${ }^{1}$, S. HIRTE ${ }^{1}$, M.J. IRWIN ${ }^{2}$ AND M. ODENKIRCHEN ${ }^{3}$ \\ ${ }^{1}$ WIP Astronomie, Universität Potsdam, Germany \\ ${ }^{2}$ RGO Cambridge, England \\ ${ }^{3}$ Sternwarte der Universität Bonn, Germany
}

From measurements of Tautenburg Schmidt plates with the APM facility in Cambridge we obtained absolute proper motions of the Galactic globular clusters M 3 and M92 directly with respect to large numbers of background galaxies (Scholz et al. 1993, 1994). We have extended our work to the dSphs in Draco and Ursa Minor (Scholz \& Irwin 1994) and to other Galactic globular clusters using Tautenburg, Palomar and UK Schmidt plates. Combining our absolute proper motion of a cluster with its known radial velocity and distance (using common parameters of the solar motion) we derive the cluster orbit in the Galaxy (cf. Odenkirchen \& Brosche 1992).

Our new results for M 12 are in good agreement with those of Brosche et al. (1991). For M5 we found an absolute proper motion in the same direction as given by Cudworth \& Hanson (1993) but only about half of the value in $\mu_{\delta}$. Our velocity values (right-handed system) differ from those given in Cudworth \& Hanson (1993) by $88 \mathrm{~km} / \mathrm{s}$ in U and $216 \mathrm{~km} / \mathrm{s}$ in V.

\begin{tabular}{lrrrrr}
\hline Cluster & \multicolumn{1}{c}{$\mu_{\alpha} \cos \delta$} & $\mu_{\delta}$ & $U$ & \multicolumn{1}{c}{$V$} \\
& \multicolumn{2}{c}{$[\mathrm{mas} / \mathrm{a}]$} & & $W$ \\
\hline M 5 & $+6.7 \pm 0.5$ & $-7.8 \pm 0.4$ & $316 \pm 31$ & $195 \pm 26$ & $-203 \pm 29$ \\
M 12 & $+3.1 \pm 0.6$ & $-7.5 \pm 0.9$ & $88 \pm 17$ & $131 \pm 31$ & $-166 \pm 24$ \\
\hline
\end{tabular}

\section{References}

Brosche, P., Tucholke, H.-J., Klemola, A.R., Ninkovic, S., Geffert, M., Doerenkamp, P. (1991), AJ, 102, pp. 2022-2027

Cudworth, K.M. and Hanson, R.B. (1993), $A J$, 105, pp. 168-172

Odenkirchen, M., Brosche, P. (1992), Astron. Nachr., 313, pp. 69-81

Scholz, R.-D., Odenkirchen, M. and Irwin, M.J. (1993), MNRAS, 264, pp. 579-586

Scholz, R.-D., Odenkirchen, M. and Irwin, M.J. (1994), MNRAS, 266, pp. 925-932

Scholz, R.-D. and Irwin, M.J. (1994), In: H.T. MacGillivray et al. (eds.), Astronomy from Wide-Field Imaging, Proc. IAU Symp. 161, pp. 535-539 